\title{
Reacción a un tatuaje de henna negra tratado con furoato de mometasona y gel de silicona. Caso clínico
}

\author{
C. M. Aboitiz-Rivera • R. Blachman-Braun • L. G. Ferrer-Arellano
}

\begin{abstract}
Reaction to a black henna tattoo treated with mometasone furoate and silicone gel: case report
\end{abstract}

Black henna tattoos have paraphenylenediamine (PPD), which contains a product of herbal origin, which due to its molecular characteristics is capable of inducing, in susceptible individuals, a type IV hypersensitivity reaction. It clinically manifests as a contact dermatitis that usually when it disappears, scarring and hypopigmentation are left in the injured area. Objective: To describe the case of a patient with hypersensitivity to henna tattoo and to present the most relevant phenomena associated with this condition. Case report: The case of a 6 year-old patient with a black henna tattoo on his right leg, who was diagnosed with contact dermatitis probably attributed to PPD, is presented. Mometasone furoate and topical silicone gel treatment was started with good response. Conclusion: Mometasone furoate and silicone gel are a good possible therapeutic option for treating contact dermatitis caused by PPD as the dermatosis was resolved without residual lesions.

(Key words: Henna, paraphenylenediamine, type IV hypersensitivity reaction, mometasone furoate, silicone gel).

Rev Chil Pediatr 2014; 85 (6): 720-723

\section{RESUMEN}

Los tatuajes de henna negra son aquellos que contienen parafenilendiamina (PPD), que contienen un producto de origen herbal, que por sus características moleculares es capaz de inducir, en individuos susceptibles, una reacción de hipersensibilidad tipo IV. Se manifiesta clínicamente como una dermatitis de contacto, que generalmente al desaparecer, persiste de manera residual una cicatriz hipertrófica e hipopigmentación en la zona lesionada. Objetivo: Describir el caso de un paciente con hipersensibilidad al tatuaje de henna, y presentar los fenómenos más relevantes asociados a esta patología. Caso clínico: Paciente de 6 años de edad, que se realizó un tatuaje con henna negra en la pierna derecha, en quien se diagnosticó posteriormente una dermatitis de contacto atribuida probablemente a la PPD. Se comenzó tratamiento con furoato de mometasona y gel de silicona con buena respuesta por vía tópica. Conclusión: El furoato de mometasona y gel de silicona son una

Recibido el 28 de agosto de 2014. Última versión aceptada el 06 de noviembre de 2014.

Carlos Manuel Aboitiz-Rivera

Servicio de Hemodinamia y Ecocardiografía, Instituto Nacional de Enfermedades Respiratorias (INER), Distrito Federal, México.

Ruben Blachman-Braun ( $\varangle$ ), Laura Graciela Ferrer-Arellano

Facultad de Ciencias de la Salud, Universidad Anáhuac México Norte, Edo. de México, México.

E-mail: rubenblach@gmail.com 
posible opción terapéutica de utilidad para tratar la dermatitis de contacto causada por el PPD, debido a que la dermatosis se resolvió sin lesiones residuales.

(Palabras clave: Henna, parafenilendiamina, reacción de hipersensibilidad tipo IV, furoato de mometasona, gel de silicona).

Rev Chil Pediatr 2014; 85 (6): 720-723

\section{Introducción}

Los tatuajes temporales de henna han sido utilizados durante siglos por diferentes culturas en África, India, Egipto y Medio Oriente ${ }^{1}$ en celebraciones religiosas y matrimoniales donde representan diferentes figuras o patrones en las extremidades de los participantes. En la actualidad esta práctica se ha popularizado en las culturas occidentales, principalmente en niños y adolescentes ${ }^{1,2}$.

La henna es un producto de origen herbal proveniente de la planta lawsonia inermis tras un proceso de desecación y pulverización de sus hojas. Posteriormente el polvo obtenido es mezclado con agua o aceite, formando una pasta que es aplicada sobre la piel para que así la zona adquiera una tonalidad café-rojiza ${ }^{2,3}$. Esto se debe a que la henna posee un ingrediente activo, un pigmento, llamado lawsona (2-hidroxi-1,4-naftoquinona), el cual tiene la capacidad de penetrar los estratos más superficiales de la piel y unirse a las proteínas, entre ellas la queratina, por lo que la tinción es temporal y va desapareciendo con la exfoliación de la capa córnea de la piel. Pese a la extensa utilización de esta sustancia, las reacciones de hipersensibilidad son poco frecuentes debido a la baja capacidad inmunogénica del lawsona ${ }^{3,4}$.

Con la intención de acelerar el proceso de desecación, aumentar su durabilidad, generar un tono más oscuro y un trazo mejor delimitado, actualmente la henna se ha mezclado con diferentes sustancias, entre ellas: limón, remolacha, cáscara de nuez, aceites esenciales y colorantes como azul índigo, añil (obtenido de la planta indigofera tinctoria) y parafenilendiamina (PPD), este último es el más utilizado en la actualidad ${ }^{5}$. Al mezclar la henna con PPD se obtiene la henna negra. La PPD es una molécula capaz de provocar, en individuos susceptibles, una reacción de hipersensibilidad tipo
IV. Las lesiones causadas por la henna negra se presentan como una dermatitis de contacto de tipo eccematoso. La reacción no suele ser inmediata, y a largo plazo puede dar lugar a cambios en la pigmentación de la piel, siendo la hipopigmentación la más frecuente en niños ${ }^{6}$, y cambios en textura de la piel que pueden llegar incluso a causar una cicatriz hipertrófica ${ }^{7}$. Pese a las importantes consecuencias estéticas de la lesión causada por la henna negra, no se ha publicado ningún consenso sobre cuál es el tratamiento más efectivo para esta condición. El objetivo de este artículo es discutir el caso de una paciente que desarrolló una reacción de hipersensibilidad al tatuaje de henna negra.

\section{Caso clínico}

Niña de 6 años de edad, que consultó consulta por dolor y prurito en miembro inferior derecho. Al interrogatorio la madre comentó que acababan de regresar de un viaje a Cancún, México, en donde la niña se realizó un tatuaje con henna negra el cual le comenzó a generar prurito a los 9 días de la aplicación (figura 1). Destaca que no es la primera vez que se realizaba un tatuaje temporal con esta sustancia y que en ocasiones previas no habían existido complicaciones, informando además que la niña no padecía ningún tipo de alergia a medicamentos, tintes, ropa o cualquier otra sustancia. No se refirió ningún otro tipo de antecedente médico de importancia.

A la exploración física se observó un placa donde se encontraba el tatuaje de henna negra de 21 días de antigüedad con bordes eritematosos, a la palpación se percibió elevación en la zona tatuada la cual se encontraba rodeada de pápulas que se adjudicaron a piquetes de mosquitos (figura 2A). Con base a lo descrito se formuló el diagnóstico de dermatitis de 


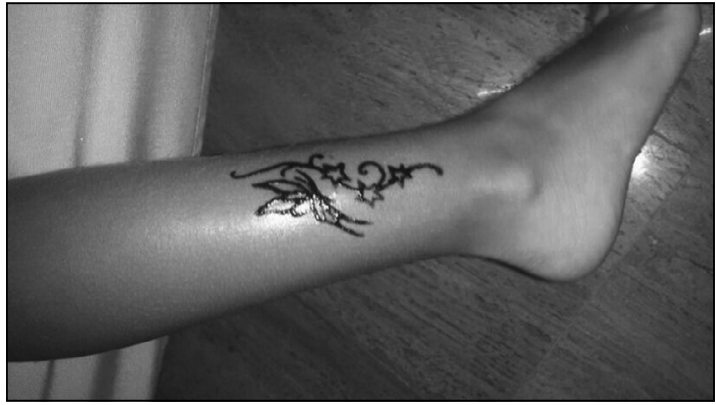

Figura 1. Imagen proporcionada por la madre de la paciente, donde se observa el tatuaje original de henna negra.

contacto causada por reacción de hipersensibilidad tipo IV, posiblemente asociada a la PPD de la henna negra.

Se inició tratamiento con ungüento de furoato de mometasona al $0,1 \%$ de manera tópica dos veces al día y gel de silicona una vez al día, con lo que disminuyó el eccema. A los 13 días del comienzo del tratamiento se observó una disminución paulatina de la inflamación local y de las molestias causadas por la reacción de hipersensibilidad (figura $2 \mathrm{~B}$ ). $\mathrm{La}$ paciente fue dada de alta a las 12 semanas de comenzar el tratamiento sin ningún tipo de lesión o alteración importante en la zona afectada (figura 2C).

\section{Discusión}

La PPD es una molécula pequeña que actúa como un hapteno, al unirse a una proteína intracelular o extracelular, generando así el complejo hapteno-proteína que tiene la capacidad de estimular al sistema inmune, principalmente a los linfocitos $\mathrm{T}$, y generar una reacción tipo IV manifestada clínicamente como una dermatitis de contacto $^{7-9}$.

A pesar de que la paciente reportada no presentó ningún otro tipo de alergia, la literatura menciona que un dato clínico de gran utilidad en relación a los pacientes con algún tipo de reacción de hipersensibilidad hacia la henna negra, es que suelen presentar reacciones cruzadas contra diferentes sustancias, esto por la similitud molecular de la PPD con colorantes utilizados en la industria textil (naranja dis-

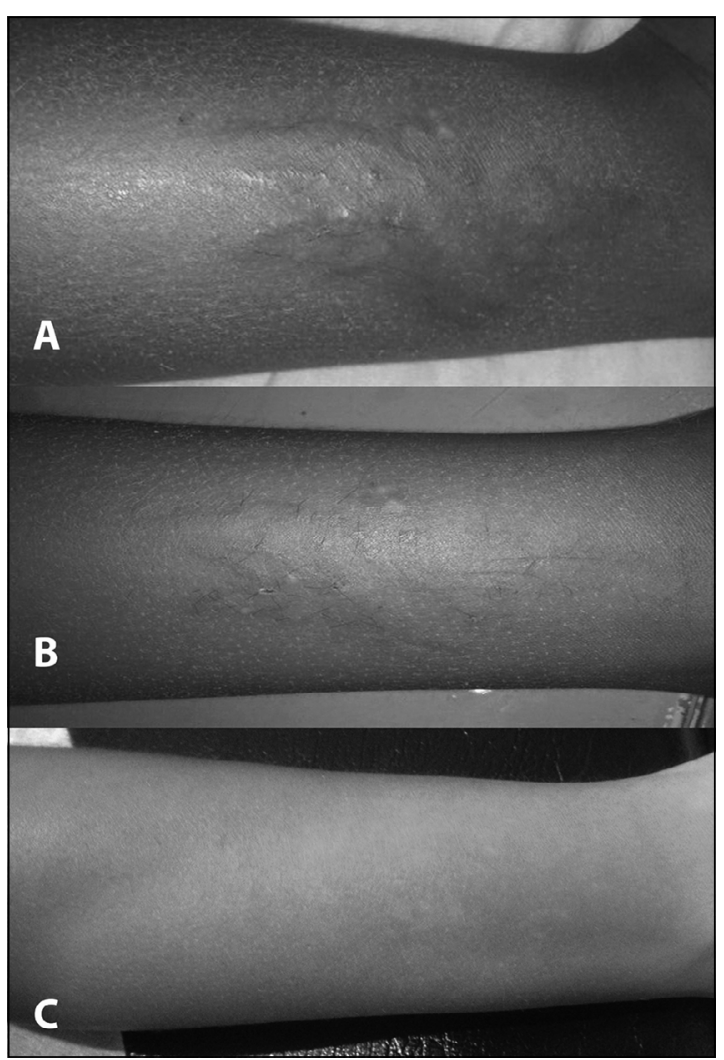

Figura 2. A. Lesión al inicio del tratamiento, la lesión permanece eritematosa con los bordes elevadas y rodeados por pápulas que se adjudican a los piquetes de mosquitos; B. Lesión a los 13 días de inicio del tratamiento, existe una disminución del eritema y de la elevación de la piel; C. Lesión a las 12 semanas de inicio del tratamiento, no existe formación de cicatriz hipertrófica y las lesiones de hipopigmentación casi imperceptibles.

perso 3, amarillo disperso 3, rojo 1, colorantes del caucho negro), cosmética (3-aminofenol, 4-aminofenol, paratoluendiamina y diaminotoluensulfato), medicamentos para la diabetes (sulfonamidas), anestésicos locales (benzocaína y procaína), derivados del ácido paraaminobenzoico (PABA) y diuréticos como la hidroclorotiazida ${ }^{10,11}$.

En la actualidad se han utilizado diversos tratamientos para pacientes con hipersensibilidad hacia la henna negra, donde los más utilizados son corticoides de moderada potencia, siendo estos por sí solos, en ocasiones, incapaces de evitar secuelas estéticas como la hipopigmentación y cicatrices hipertróficas en la 
zona de la lesión ${ }^{5-7,9}$. Debido a ello se decidió utilizar un tratamiento a base furoato de mometasona y gel de silicona, este último con la intención de disminuir las lesiones cutáneas residuales.

Se observó mejoría sustancial de la lesión original pero persistieron las lesiones de hipopigmentación aunque aparentemente fueron menores a las reportadas en la literatura ${ }^{2,7}$. Esto lo podemos adjudicar a las características farmacocinéticas y farmacodinámicas del gel de silicona, entre las que destacan: el aumento en la hidratación del estrato corneo, facilitación en la regulación de los fibroblastos y disminución en la producción de colágena, regulación de factores de crecimiento (TGF $\beta$ y FGF $\beta$ ), reducción de prurito y molestias en la zona. Podemos considerar que el uso de gel de silicona puede ser útil para evitar la aparición de la cicatrices hipertróficas, razón por la que, probablemente, la paciente no presentó cicatriz hipertrófica residual ${ }^{11}$. También consideramos que las características farmacodinámicas y el potencial antiinflamatorio de los esteroides tópicos como el furoato de mometasona, un esteroide tópico de baja absorción y potencia media, probablemente contribuyeron al buen pronóstico de la paciente, pese a lo que en la literatura se ha utilizado poco para el tratamiento de esta patología ${ }^{12}$.

\section{Conclusión}

Las reacciones de hipersensibilidad causadas por la henna negra cada vez serán más frecuentes, debido a la creciente popularización de esta práctica, por esta razón el médico debe ser capaz de reconocer de manera oportuna esta patología. Consideramos que la administración de furoato de mometasona y gel de silicona por vía tópica, podrían ser una buena opción terapéutica para la dermatitis de contacto ocasionada por una reacción cutánea de hipersensibilidad tipo IV contra la PPD. Creemos que se debe obtener más experiencia con esta combinación terapéutica, debido a que los resultados observados en este caso fueron de gran beneficio para la paciente.
Potenciales conflictos de interés: Este trabajo cumple con los requisitos sobre consentimiento/asentimiento informado, comité de ética, financiamiento, estudios animales y sobre la ausencia de conflictos de intereses según corresponda.

\section{Referencias}

1.- Rahmoun $M N$, Benabdallah $M$, Villemin D, et al: Antimicrobial screening of the Algerian Lawsonia inermis (henna). Der Pharma Chemica 2010; 2: 320-6.

2.- Silvente C, Valdivielso M, De la Cueva P, Balbín E, Chavarría E, Hernanz JM: Dermatitis alérgica de contacto tras un tatuaje con henna. Acta Pediatr Esp 2011; 69: 75-8.

3.- Lasa EM, Cojocariu Z, Arroabarren E, Echechipia S, Marín MPL, Tabar AI: Tatuaje de henna en niños: ¿natural y temporal?. An Sist Sanit Navar 2007; 30: 131-4.

4.- Almeida PJ, Borrego L, Pulido-Melián E, GonzálezDías $O$ : Quantification of p-phenylenediamine and 2-hydroxy-1,4-naphthoquinone in henna tattoos. Contact Dermatitis 2011; 66: 33-7.

5.- Arranz DM, Corral M, Vidaurrázaga Díaz C, De Lucas $R$, Díaz R: Riesgos de los tatuajes de henna negra. An Pediatr (Barc) 2005; 63 (5): 448-52.

6.- Sonnen G: Type IV hypersensitivity reaction to a temporary tattoo. Proc (Bayl Univ Med Cent) 2007; 20: 36-8.

7.- Vasilakis V, Knight B, Lidder S, Frankton S: Severe type IV hypersensitivity to 'black henna' tattoo. BMJ Case Reports. 2010.

8.- Elliott G, Das PK: Defining the Antigen Determinant for T-Cell-Mediated Contact Dermatitis Using p-Phenylenediamine: A Gateway to Chemical Immunology. J Invest Dermatol 2010; 130: 641-3.

9.- Ong GY: Temporary Tattoo Associated Type IV Delayed Hypersensitivity Dermatitis in a Child-A Case Report and Call for Parental Caution in Singapore. Ann Acad Med Singapore 2010; 39: 738-42.

10.- Sánchez AI, Gatica ME, García D, Larralde M: Dermatitis alérgica de contacto por tatuajes temporales de "henna negra". Arch Argent Pediatr 2010; 108: e96-9.

11.- Puri N, Talwar A: The efficacy of silicone gel for the treatmet of hypertrophic scar and keloids. J Cutan Aesthet Surg 2009; 2: 104-5.

12.- Duk K, Hyun L, Chun P, et al: The Clinical Efficacy of Mometasone Furoate in Multi-Lamellar Emulsion for Eczema: A Double-blinded Crossover Study. Ann Dermatol 2013; 25: 17-22. 